\title{
An interesting consequence of the Heisenberg construction
}

B. Sury

B. Sury got his Ph.D. degree from the Tata Institute of Fundamental Research in Mumbai (Bombay) in 1991. In 1999, he moved from the Tata Institute to the Indian Statistical Institute in Bangalore. His interests are in group theory and number theory. $\mathrm{He}$ is also involved in the mathematical olympiad programme in his country.

The projective linear groups over finite fields give families of nonabelian finite simple groups and it is natural to wonder as to which groups are of the form $G / Z(G)$ for some group $G$, where $Z(G)$ denotes the center of $G$. In fact, ideally one would like to characterize all of them. It is natural to believe such a characterization could probably be obtained from the theory of central extensions. Indeed, these groups have been studied under the name of capable groups for some time now. Let us first think about the (far) simpler problem of finite abelian groups which are of this form. The answer turns out to be simple but interesting. Before stating the result, let us notice that the question is about 2-step nilpotent groups, since $G / Z(G)$ is nontrivial, abelian if and only if $G$ is 2-step nilpotent.

To exemplify the result, let us look at the example of the quaternion group

$$
H=\{ \pm 1, \pm i, \pm j, \pm k\}
$$

Nach Einführung des Gruppenbegriffs in der Algebra besteht eine der ersten Aufgaben darin, Gruppen kleiner Ordnung zu klassifizieren. Beispielsweise stellt Serge Lang in seinem Standardwerk zur Algebra die Aufgabe, alle Gruppen der Ordnung kleiner oder gleich 10 bis auf Gruppenisomorphie zu bestimmen. Dazu werden Struktursätze herangezogen, die am Anfang der Gruppentheorie stehen. Einer dieser Sätze besagt, dass unter der Voraussetzung der Zyklizität der Faktorgruppe $G / Z(G)$ von $G$ nach dem Zentrum $Z(G)$ die Gruppe $G$ selbst abelsch ist. In der vorliegenden Arbeit wird eine Variation dieser Fragestellung untersucht. Der Autor stellt sich die Frage, welche endlichen abelschen Gruppen $A$ in der Form $G / Z(G)$ auftreten, wobei das Zentrum $Z(G)$ zyklisch ist (und die Gruppe $G$ nicht mehr endlich zu sein braucht). Er beweist unter diesen Voraussetzungen, dass die Isomorphie $A \cong B \times B$ mit einer abelschen Gruppe $B$ besteht. Insbesondere kann eine endliche abelsche Gruppe $A$ nicht von der Form $G / Z(G)$ sein, wenn die Ordnung von $A$ keine Quadratzahl ist. 
with the multiplication rules $i j=k=-j i, i^{2}=j^{2}=k^{2}=-1$. Note that its center $Z$ is $\{ \pm 1\}$. One has $H / Z \cong \mathbf{Z} / 2 \mathbf{Z} \times \mathbf{Z} / 2 \mathbf{Z}$. Indeed, one isomorphism is given by mapping \pm 1 to $(0,0), \pm i$ to $(1,0), \pm j$ to $(0,1)$ and $\pm k$ to $(1,1)$.

\section{Theorem.}

(a) Let $A$ be a finite abelian group which is isomorphic to $G / Z(G)$ for some (possibly infinite) group $G$ whose center $Z(G)$ is cyclic. Then $A \cong B \times B$ for some abelian group $B$. In particular, finite abelian groups of non-square order can never be expressed as $G / Z(G)$ for some $G$ with cyclic center $Z(G)$.

(b) Conversely, let $A$ be a finite abelian group. Then, the additive group $A \times A^{*}$ is isomorphic to $G / Z(G)$ for some group $G$. Here $A^{*}=\operatorname{Hom}\left(A, S^{1}\right)$, the character group.

(c) There exist abelian groups of non-square orders which are of the form $G / Z(G)$ with the center $Z(G)$ non-cyclic.

Proof. (a) Firstly, suppose that $G$ is any finite group such that $G / Z(G)$ is abelian. We write $Z$ in place of $Z(G)$ for simplicity as there will be no confusion here. Then, the commutator map $c: G \times G \rightarrow G$ maps into $Z$. Moreover, it clearly induces a map (again denoted by $c$ ) from $G / Z \times G / Z$ to $Z$. Using the fact that the values are in the center $Z$, it follows that $c: G / Z \times G / Z \rightarrow Z$ is bilinear. Moreover, $c$ is non-degenerate because if $c(x Z, y Z)=1$ for all $y Z \in G / Z$, then $x \in Z$; that is, $x Z$ is the identity in $G / Z$. We note that $c$ is evidently skew-symmetric. Now, as $Z$ is cyclic, we may take a character of it into $S^{1}$; the corresponding bilinear form is again non-degenerate. Then, from the theory of bilinear forms, one has $G / Z \cong M \times M$ for a maximal totally isotropic subgroup $M$ of $G / Z$. Note that $M=A / Z$ where $A$ is a maximal abelian subgroup of $G$ containing $Z$. Thus, any abelian group isomorphic to $G / Z$ with finite $G$ which has cyclic center $Z$, must be isomorphic to $M \times M$ for some group $M$.

To complete the proof of part (a), we need to show that if a finite abelian group $A$ is of the form $G / Z(G)$ for some group $G$, then it is also isomorphic to a group of the form $F / Z(F)$ where $F$ is finite and, further, if $Z(G)$ is cyclic, then we can choose $F$ with $Z(F)$ cyclic. To see this, we will observe first that $G$ can be assumed to be finitely generated. Writing $A=G / Z(G)=\left\{g_{1} Z(G), \ldots, g_{n} Z(G)\right\}$, we take $G_{1}$ be the subgroup of $G$ generated by $g_{1}, \ldots, g_{n}$. Note that $G_{1} \cdot Z(G)$ is a subgroup of $G$ and $G=\bigcup_{i=1}^{n} g_{i} Z(G) \subseteq G_{1} \cdot Z(G)$ which means $G=G_{1} \cdot Z(G)$. Now, each element of $Z\left(G_{1}\right)$ commutes with any element of $G_{1}$ as well as with any element of $Z(G)$ and, therefore, with the whole of $G_{1} \cdot Z(G)=G$ itself. In other words, $Z\left(G_{1}\right) \leq Z(G)$ and hence $Z\left(G_{1}\right)=Z(G) \cap G_{1}$. Hence, we have

$$
G / Z(G)=G_{1} \cdot Z(G) / Z(G) \cong G_{1} /\left(Z(G) \cap G_{1}\right)=G_{1} / Z\left(G_{1}\right) .
$$

Note also that if $Z(G)$ is cyclic, then so is $Z\left(G_{1}\right)=Z(G) \cap G_{1}$. Thus, we may replace $G$ by $G_{1}$ and assume that $G$ is finitely generated. Now, since $Z(G)$ is of finite index in $G$, it is a finitely generated abelian group. Writing $Z(G)=Z_{\text {tor }} Z_{0}$ where $Z_{0}$ is the free abelian part and $Z_{\text {tor }}$ is the torsion part, we look at the finite group $H=G / Z_{0}$. We have $[G, G] \leq Z(G)$ as $G / Z(G)$ is abelian. But in any group $G$ where $Z(G)$ has finite index $n$, the commutator subgroup $[G, G]$ is a finite $n$-torsion group by a famous theorem due to 
Schur. Indeed, in the special case we have considered, viz., when $G / Z(G)$ is abelian, this is far easier as we show now. Let $n$ denote the order of the abelian group $G / Z(G)$. So, $[G, G] \leq Z(G)$. Also, $g^{n} \in Z(G)$ for any $g \in G$ and, we have

$$
\left(x y x^{-1}\right)^{n}=x y^{n} x^{-1}=y^{n} \quad \forall x, y \in G .
$$

Using this and the fact that $[G, G] \leq Z(G)$, we have for any $x, y \in G$ that

$$
y^{n}=\left(x y x^{-1}\right)^{n}=\left(x y x^{-1} y^{-1} y\right)^{n}=\left(x y x^{-1} y^{-1}\right)^{n} y^{n}
$$

which implies

$$
\left(x y x^{-1} y^{-1}\right)^{n}=1 .
$$

As $[G, G]$ is abelian and is generated by commutators, we have $u^{n}=1$ for all $u \in[G, G]$. But, $[G, G]$ is a finitely generated abelian group - it can be generated by the commutators $t_{i} t_{j} t_{i}^{-1} t_{j}^{-1}$ where $G=\bigcup_{i=1}^{n} Z(G) t_{i}$. Hence $[G, G]$ is a finite, abelian, $n$-torsion group.

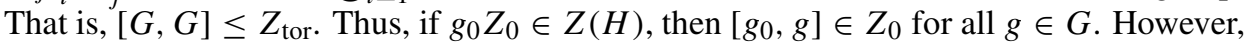
$\left[g_{0}, g\right] \in Z_{\text {tor }}$; hence $\left[g_{0}, g\right]=1$ and so $g_{0} \in Z(G)$. Hence the coset $g_{0} Z_{0} \in z Z_{0}$ for some $z \in Z_{\text {tor. }}$ Conversely, every coset in $H$ of the form $z Z_{0}$ with $z \in Z_{\text {tor }}$ is in $Z(H)$. Therefore, $Z(H)=Z\left(G / Z_{0}\right)=Z(G) / Z_{0} \cong Z_{\text {tor }}$. Thus, $A \cong H / Z(H)$. Further, clearly if $Z(G)$ is cyclic, then so is $Z(H) \cong Z(G) / Z_{0}$. This completes the proof of part (a).

(b) Conversely, for any finite abelian group $A$, look at the group $G=A \times A^{*}$. If $A$ has order $n$, then we have a map $f: G \times G \rightarrow \mu_{n}$ given by

$$
f\left((a, \chi),\left(a^{\prime}, \chi^{\prime}\right)\right)=\chi\left(a^{\prime}\right) .
$$

Here, $\mu_{n}$ is the subgroup of $n$-th roots of unity in $S^{1}$. Note that $f$ satisfies the so-called 2-cocycle condition

$$
f\left(g, g^{\prime}\right) f\left(g g^{\prime}, g^{\prime \prime}\right)=f\left(g^{\prime}, g^{\prime \prime}\right) f\left(g, g^{\prime} g^{\prime \prime}\right)
$$

because $\chi\left(a^{\prime}\right)\left(\chi \chi^{\prime}\right)\left(a^{\prime \prime}\right)=\chi^{\prime}\left(a^{\prime \prime}\right) \chi\left(a^{\prime} a^{\prime \prime}\right)$ for each $a, a^{\prime}, a^{\prime \prime} \in A$ and $\chi, \chi^{\prime} \in A^{*}$. Now, let us consider the set $\tilde{G}:=G \times \mu_{n}$ as a group under the multiplication

$$
(g, x)\left(g^{\prime}, x^{\prime}\right):=\left(g g^{\prime}, x x^{\prime} f\left(g, g^{\prime}\right)\right) .
$$

Note that associativity is precisely the 2-cocycle condition satisfied by $f$. The identity element is $(1,1)$ and

$$
(g, t)^{-1}=\left(g^{-1}, t^{-1} f\left(g, g^{-1}\right)^{-1}\right)=\left(g^{-1}, t^{-1} \chi(a)\right)
$$

where $g=(a, \chi)$. Evidently, $\mu_{n}$ sits as a subgroup of $\tilde{G}$ by the second inclusion because $f(1,1)=1$; we claim that it is the center of $\tilde{G}$. Indeed, $((a, \chi), t)$ is in the center if and only if, $\chi\left(a^{\prime}\right)=\chi^{\prime}(a)$ for all $a^{\prime} \in A, \chi^{\prime} \in A_{\tilde{G}}^{*}$. This forces $a=1$ in $A$ and $\chi=1$ in $A^{*}$ and $t$ is arbitrary. Hence, we have that $G \cong \tilde{G} / \mu_{n}$. The proof of part (b) is complete.

(c) Let $F$ be a finite field and $E \subset F$ be a proper subfield. Consider the group

$$
G=\left\{\left(\begin{array}{ccc}
1 & a & c \\
0 & 1 & b \\
0 & 0 & 1
\end{array}\right): b, c \in F ; a \in E\right\} .
$$


Note that if we denote a typical element of $G$ by $g(a, b, c)$, then

$$
g(a, b, c) g\left(a^{\prime}, b^{\prime}, c^{\prime}\right)=g\left(a+a^{\prime}, b+b^{\prime}, a b^{\prime}+c+c^{\prime}\right) .
$$

Note $g(a, b, c)^{-1}=g(-a,-b, a b-c)$. Now, $g(a, b, c) \in Z(G)$ if and only if $a b^{\prime}=a^{\prime} b$ for all $a^{\prime} \in E, b^{\prime} \in F$. Thus, some $g(a, 0, c) \in Z(G)$ if and only if $a b^{\prime}=0$ for all $b^{\prime} \in F$; that is, if and only if $a=0$. On the other hand, if some $g(a, b, c) \in Z(G)$ with $b \neq 0$, then $g(a, b, c) g(1,0,0)=g(1,0,0) g(a, b, c)$ gives $0=b$, a contradiction. Thus, $Z(G)=\{g(0,0, c): c \in F\}$. Note that $G / Z(G) \cong E \oplus F$ which can have non-square order (for instance, if $E=\mathbf{F}_{p}, F=\mathbf{F}_{p^{2}}$. We finally note that $Z(G)$ is not cyclic. The proof of (c) is complete.

\section{An example.}

In the proof of part (a) of the theorem, we used the cyclicity of $Z$ and the existence of nondegenerate alternating bilinear forms to conclude that the group has square order. This can fail in general if $Z$ is not cyclic. For instance, for any prime $p$, there is a non-degenerate alternating bilinear form on $(\mathbf{Z} / p \mathbf{Z})^{3}$ with values in $(\mathbf{Z} / p \mathbf{Z}) \times(\mathbf{Z} / p \mathbf{Z})$. This is because a bilinear form with values in $(\mathbf{Z} / p \mathbf{Z}) \times(\mathbf{Z} / p \mathbf{Z})$ is a pair of forms with values in $\mathbf{Z} / p \mathbf{Z}$. If we take a pair of alternating forms on $(\mathbf{Z} / p \mathbf{Z})^{3}$ whose null spaces are different, we get an example.

\section{Remarks.}

(a) In fact, an argument given in the theorem can be generalized to prove (see [1]) that any finite group of the form $G / Z(G)$ is isomorphic to one of the form $H / Z(H)$ where $H$ is finite.

(b) The allusion to the Heisenberg group in the title can be explained briefly as follows. If $\Omega$ is a non-degenerate alternating form on a real vector space $V$ (which must thus necessarily have some even dimension $2 n$ ), recall that the corresponding Heisenberg group $H(V, \Omega)$ is defined as the set $V \times \mathbf{R}$ under the operation

$$
(v, s)(w, t)=\left(v+w, s+t+\frac{1}{2} \Omega(v, w)\right) .
$$

The center of this group is $Z:=\{(0, t): t \in \mathbf{R}\}$ and the quotient $H(V, \Omega) / Z \cong$ $\mathbf{R}^{n} \times \widehat{\mathbf{R}^{n}}$ as Lie groups. The group $\widehat{\mathbf{R}^{n}}$ on the right side is the Pontryagin dual group of $\mathbf{R}^{n}$ (and hence isomorphic to it). Our result above shows that this is essentially the only way to produce analogously a finite abelian group of the form $G / Z(G)$ for some group $G$. In an epoch-making paper ([2]), André Weil uses the action of the symplectic group of $(V, \Omega)$ on the Heisenberg group to define a projective representation, the so-called Weil representation. In particular, he extends this construction to the situation where $\mathbf{R}^{n}$ is replaced by any group isomorphic to its Pontryagin dual. These developments have not only allowed us to do harmonic analysis on locally compact groups which arise in number theory but have also given a representationtheoretic description of theta functions. 
Acknowledgement. It is a pleasure to thank Dipendra Prasad who pointed out to me that the correct way to view the observation of the theorem is as a very special case of Weil's ideas centred around the Weil representation. I also thank him for the example given above. Further, I am grateful to an anonymous referee's remarks which among other things urged me to simplify the original proof of theorem (a) where I had used Schur's result.

\section{References}

[1] Isaacs, M.: Derived subgroups and centers of capable groups. Proc. Amer. Math. Soc. 129 (2001), 28532859.

[2] Weil, A.: Sur certains groupes d'opérateurs unitaires. Acta Math. 111 (1964), 143-211.

B. Sury

Stat-Math Unit

Indian Statistical Institute

8th Mile Mysore Road

Bangalore 560 059, India.

e-mail: sury@isibang.ac.in 